\title{
Investigating cloud ERP challenges in public universities in the Middle East "Field Study"
}

\author{
Hussain Ahmad Awad, Fadi Mohammed Batta \\ MIS department, Faculty of Business - Khulais, King Abdulaziz University, Jeddah, KSA
}

Email address:

haawad@kau.edu.sa (H. A. H. Awad), fbattah@kau.edu.sa (F. M. Batta)

\section{To cite this article:}

Hussain Ahmad Awad, Fadi Mohammed Batta. Investigating Cloud ERP Challenges in Public Universities in the Middle East "Field Study". Internet of Things and Cloud Computing. Vol. 2, No. 3, 2014, pp. 12-16: 10.11648/j.iotcc.20140203.11

\begin{abstract}
ERP is a concept that has been around for some time, and such as any other field; it is inevitable for ERP to find new ways to cope with the huge changes in Information Systems world. One of the new ideas was to facilitate ERP through cloud computing systems; in order to fulfil the staggering raise in service requests from ERP customers due to infrastructure and technology advancement. Google, SAP, INFOR and other ERP service providers has already adopted the idea of providing ERP services through the cloud, in order to attract more business, in particular the more expanding and demanding segment of small and medium enterprises (SME's). We, on the other hand would like to study the challenges and opportunities of cloud ERP in educational enterprises; public universities in specific. Hussain (7) has mentioned these challenges; we will expand that by validating the challenges alongside providing an ordered view of them, a survey amongst wide sample of public universities in the Middle East will investigate further challenges that could ascend along application of Cloud ERP, and classify them in orderly fashion. Which eventually we think it will help public universities to further more understand how to benefit from cloud ERP.
\end{abstract}

Keywords: Enterprise Resource Planning (ERP), Cloud ERP Services, Challenges of ERP, Cloud in Public Universities, Public Schools (Universities) and ERP Systems

\section{Introduction}

Organizations - including universities- seek to facilitate their operations and supply chain management; one of most widespread solution in the modern age is the Enterprise Resource Planning systems, Staehr defines ERP as "ERP systems are integrated software packages with a common database that support business processes in companies"(17).

ERP has shown many benefits on organizations, such as cost reduction, improving decision-making, increase efficiency, operation flexibility and other benefits that all work together to sustain the organization position among its peer and achieve success. Yet, ERP is a system, like any system; the more the organization components are divergence and interconnected, the more the system is complicated accordingly. This may inflict more issues in case of new ERP adoption; since ERP is a system that will merged with all organization operations and structure. To make sure that this merger is healthy and successful; the need for and efficient IT staff is crucial to manage and apply such changes. All of that will dramatically will raise many issues in ERP application in organization, the major challenge of adopting these systems is the cost of implementing and maintenance which must be always evaluated by return of investment (ROI) calculation .(8). These costs depend on organizational size, users numbers, processes complexity, system boundaries, and system scope.(14) also the mentioned factors must be taken in mind upon preparing the infrastructure accommodate new system in terms of hardware, software, training, networks, etc..(11).

Keeping all that in mind, ERP providers start looking for new areas to enhance ERP in order to manage these challenges; that when cloud made its way as a new operations model for ERP system to help providers to cope with new and commanding challenges.

\section{Cloud and ERP}

Cloud computing model is a new technology that mainly aim to provide application and system services for clients without need for hardware, software and networks, but through using of provider portal.(2) 
Since cloud computing has forced itself as a new operation model that can be used by ERP and other systems; organization looking for new options started considering this trend seriously; on the other, hand vendors such as Oracle, Google, Microsoft, DATABIS and others have worked to provide modern system services through cloud computing model including ERP systems. those vendors based there work on the expectations that cloud will be option number one for organizations looking for advanced modern services; small and medium enterprises (SME) also showed increasing interest in such model, since infrastructure costs are no longer needed.(18)

Vendors provided cloud services through contractual periodical payments, The amount of payment depends on the type of service, number of license (users), and data flow by customer.(5)

ERP providers have three operational models of Cloud ERP to their customers, choosing one of them identify how the provider and customer manages their relation. Margaret Rouse defines these operational models as:

"Software as a Service (SaaS) is a software distribution model in which applications are hosted by a vendor or service provider and made available to customers over a network, typically the Internet". (12)

"Infrastructure as a Service (IaaS) is a provision model in which an organization outsources the equipment used to support operations, including storage, hardware, servers and networking components. The service provider owns the equipment and is responsible for housing, running and maintaining it. The client typically pays on a per-use basis". (12)

"Platform as a Service (PaaS) is a way to rent hardware, operating systems, storage and network capacity over the Internet. The service delivery model allows the customer to rent virtualized servers and associated services for running existing applications or developing and testing new ones". (12)

Many of organizations that adopt cloud computing focus on supporting applications such as office suits or small applications for daily processes, yet did not use the full capacity of cloud services to support main organization applications such as ERP or CRM (16)

\section{Cloud ERP Adoption Challenges}

Although cloud computing is available for many activities and application, with many vendors - major and small entering this field, and despite many foreseen advantages of these systems that has been discussed in literature; many organizations still prefer their own in house system especially public schools.

The authors used Hussain (7) as a background for their study, were it defined many challenges that face organizations upon cloud ERP adoption. The writers conducted a survey seeking to confirm those challenges with focus on public schools that have working in house ERP system. The authors aim to identify any other challenges in addition to ones mentioned in Hussain (7) that propose obstacles in public schools adoption for cloud ERP.

125-survey form were handed to employees and specialists in managing and running organizational systems in 15 public schools in the middle east, 100 form were retrieved and used for this study. This has added a new group of challenges to the ones mentioned in earlier study, the authors divided all challenges into two main categories, administrative and technical challenges as follows:

\subsection{Technical Challenges}

\subsubsection{Security and Privacy}

This is main challenge that face the cloud now; also, the main reason behind hesitation in cloud adoption in organization of any type. The fear on sensitive and private information can be referred to two reasons, one: organization information is exposed to the vendor, two: safety of these information rely heavily on the security of the vendor services itself against all sorts of malicious attacks.

Although vendors are not saving any resources provide tools and security systems to enhance cloud safety; internal threats impose themselves as a high risk in parallel with external threats, miss use and employee's technical ignorance are main issues that vendors must seriously address.

\subsubsection{Legacy Systems}

Active organizations rely mostly on their in house systems and application; to consider system update to cloud, organizations must keep in mind private information that could go back for long periods, this issue is already a challenge in in house migration to new system, with the cloud, this issue is more profound. . The problem arise from the different database, rules, report and data structure. (6)

\subsubsection{Implementation Challenges}

Cloud aims to provide agility and flexibility far beyond traditional systems; requirements modifications, license numbers and other elements of the system are bound to change all the time as per user's requests, cloud vendors must accommodate the cloud to be flexible accordingly. Other issue is the vast platforms and applications available for users to access the cloud services customers may use tools as PC, TAP, smart phones with different browsers. (9)

\subsubsection{Compatibility Challenges}

Flexible services may not always compatible with different requirements by different customers; since differences vary between skills, objects and goals of each customer. Vendors are required to expect such variations between customers and provide more coherence with the cloud services as possible. ; Because they are described as one size fits all. (10)

\subsubsection{Availability Challenges}

One of the main concepts of cloud computing is service anywhere anytime, providers may need to interrupt that idea sometimes when making system updates or new tools installation, which may cause service delay or denial at some point. 


\subsubsection{Integration Level Challenges}

Customers of cloud ERP will start providing their services after adoption, yet some of old system components may be required to stay out of the cloud, which could raise the question; will the vendors be able to integrate new services with the components that the customers opted to stay in house?

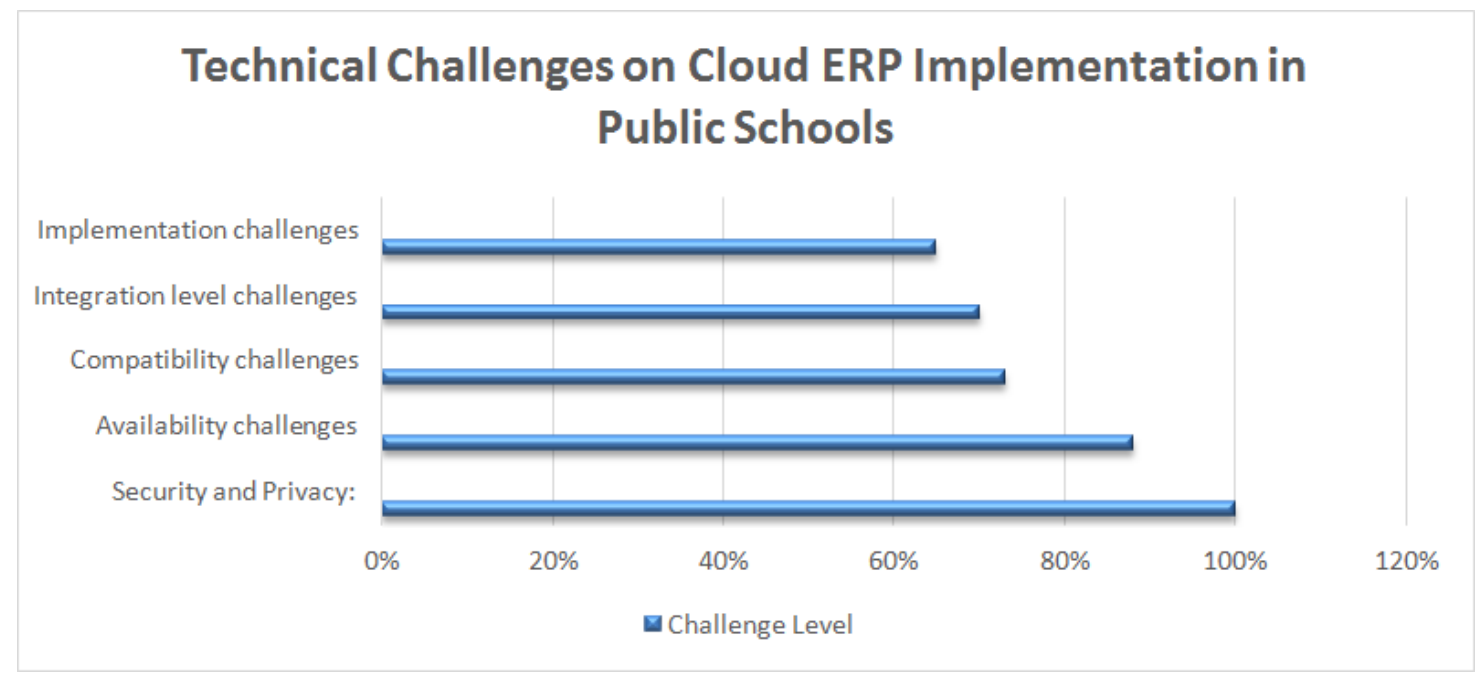

Figure 1. The following graph shows the results of the survey in relation with technical challenges of Cloud ERP implementation in public schools.

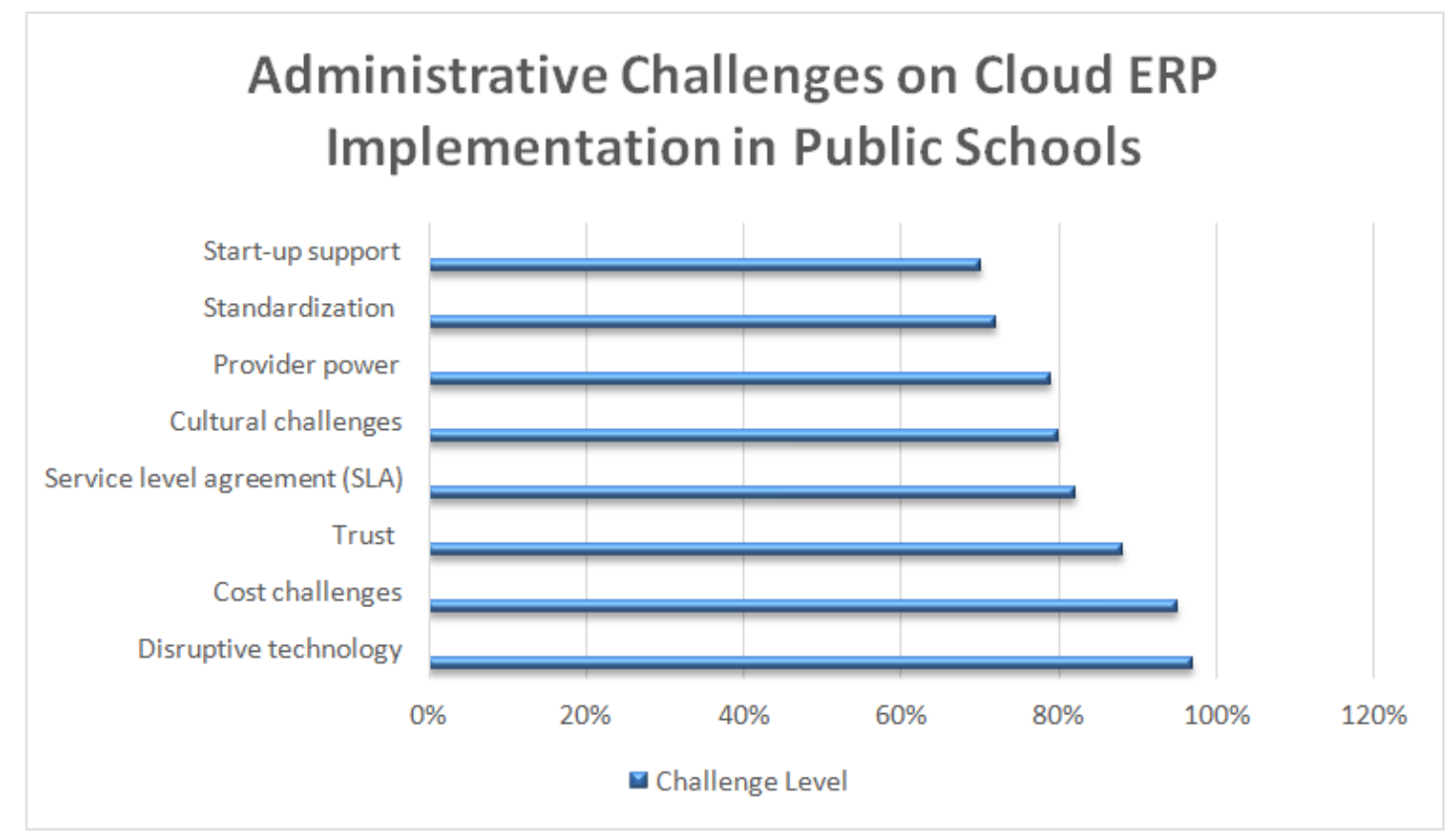

Figure 2. The following graph shows the results of the survey in relation with administrative challenges of Cloud ERP implementation in public schools

\subsection{Administrative Challenges}

\subsubsection{Disruptive Technologies}

Since cloud is a new technology; customers interested in cloud ERP implementation wish to evaluate other organizations experience with that implementation, Most cloud ERP users are SME's, which not may be a big indications for larger organizations to take such step. (4)

\subsubsection{Service Level Agreement (SLA)}

Contract or SLA between cloud ERP providers and customers govern the service level, scope, and boundaries. Customers would investigate contract conditions, and what will happen if they need to modify any level of service or other criteria such as number of licenses, bandwidth etc... (13)

\subsubsection{Cost Challenges}

All past researches promoted cloud services through the cost reduction benefit (12), yet those benefits considered in case the organization does not own in house systems or in case of owned systems; they are obsolete. However, for organizations that own working systems, facilities and infrastructure, it will need further investigation on how cloud ERP will be financially viable in comparison with existing systems. 


\subsubsection{Provider Power}

The provider will gain power of owner on information upon agreement, also information access and control will be under provider authority. In such case, what are the consequences if the contract is not renewed or cancelled? What will happen to the information in such case? In addition, is the contract infinite?

\subsubsection{Cultural Challenges}

Fear of change is one of the main issues managers needs to address; especially if that change will affect their authorities and responsibilities. Resistance to change will show in other sectors upon adopting cloud ERP or any other new system, mainly the effected parties will be the first to resist, such as IT department, who will feel obsolete upon using new system that render IT infrastructure, personnel and components unnecessary.

\subsubsection{Trust}

"Building an IT organization's confidence in a solution requires a combination of consistent performance, verifiable results, service guarantees, transparency, and plans for contingencies"(3). lack of confidence can be caused by two factors; one: the importance of the information held by cloud ERP service provider, two: Cloud ERP is still not wide spread among public schools worldwide; so yet there no formal practice that could govern such big step of migration.

\subsubsection{Standardization}

Most of cloud ERP providers are originally ERP providers, and even so there is fierce competition is this field; formal laws from governments and regulating bodies is still vague and not complete (8).

\subsubsection{Start-Up Support}

Moving to cloud has its own issues, that are directly related to the customer's nature of operations and structure, the more complicated the operations are, the more complicated the migration will be. Vendor responsibility is ti provide tools and methods to make such migration as smooth as possible.

\section{Summary}

It is well clear that any organization can benefit from the Cloud, but as for universities and public schools; hesitation is a major drawback in this sector to adopt cloud ERP. Even though educational organizations started to use cloud application such google docs and others, still there are many challenges to adopt cloud computing as an operational model for ERP services.

This paper collected challenges faced by public schools looking to adopt cloud ERP through survey, the survey confirmed the mentioned challenges previously (Hussain), and added other challenges discovered from the field study. The challenges divided into

Technical: Security and Privacy, Legacy system,
Implementation challenges, Compatibility challenges, Availability challenges, Integration level challenges.

Administrative: Disruptive technology, Service level agreement (SLA), Cost challenges, Provider power, Cultural challenges, Trust, Standardization, and Start-up support challenges

In order to facilitate cloud ERP; both vendors and customers must work together to overcome these challenges.

\section{References}

[1] Ahmed Al-Johani, Ahmed Youssef, A FRAMEWORK FOR ERP SYSTEMS IN SME BASED ON CLOUD COMPUTING TECHNOLOGY. International Journal on Cloud Computing: Services and Architecture, Vol. 3, No. 3, 2013

[2] Babcock, Charles, Why 'Private Cloud' Computing Is RealAnd Worth Considering. InformationWeek, may 11,2014, http://www.informationweek.com/news/software/hosted/show Article.jhtml?articleID=216500083.

[3] Drue Reeves et al., Cloud Computing: Transforming IT. Burton group research report, pp 33, 2009

[4] Duncan Waga, Esther Makori, Kefa Rabah, Utilization of Cloud Computing in Education and Research to the Attainment of Millennium Development Goals and Vision 2030 in Kenya. Universal Journal of Educational Research, Vol. 2, No.2, 2014

[5] Elias Kiadehi, Shahriar Mohammadi, Cloud ERP: Implementation of Enterprise Resource Planning Using Cloud Computing Technology. Journal of Basic and Applied Scientific Research Vol. 2, No. 11, 2012

[6] Everest Group research, Enterprise cloud adoption survey 2013: summary of results, cloud connect, march 2013

[7] Hussain Awad, Cloud computing as an operational model for ERP services

[8] "Definitions and challenges". International Journal of Innovation and Applied Studies IJIAS, Vol. 8, No. 4, 2014

[9] Hussain Awad, Fadi Battah, Is the Cloud Educational Enterprise Resource Planning the Answer to Traditional Educational Enterprise Resource Planning Challenges in Universities? Computer Engineering and Intelligent Systems, Vol. 3, No.6, 2012

[10] Jonathan. G, 2012 To Cloud Or Not To Cloud: That Is The Question For ERP. MHD Supply Chain Solutions, Vol. 42 Issue 1, 2012

[11] Liz Herbert, Chris Andrews, Kelsey Stone, SAP In The Cloud: Market Update And Lessons Learned From Early Adopters. Forrester Research, 2011

[12] Makkar, MEENAKSHI, EAAS -ERP AS A SERVICE. Journal of Information and Operations Management, Vol. 3, No. 1, pp141 - 145, 2012.

[13] Margaret Rouse, definition of SaaS, PaaS, and IaaS. Techtarget, search cloud computing, may 20,2014, $\mathrm{http}: / /$ searchcloudcomputing.techtarget.com/definition/Softwareas-a-Service 
[14] Marko Mijač, Ruben Picek, Zlatko Stapić, Cloud ERP System Customization Challenges. Central European Conference on Information and Intelligent System, pp 132 - 296, Croatia, 2013

[15] Petra Schubert, Femi Adisa, Cloud Cloud Computing for Standard ERP Systems: Reference Framework and Research Agenda. Fachbereich Informatik No. 16/2011.

[16] Purohi, Jaiswa, Surabhi Pandey, Challenges Involved in Implementation of ERP on Demand Solution: Cloud Computing. International Journal of Computer Science Issues, Vol. 9, Issue 4, No 2, 2012
[17] Spencer Arnesen, Is a Cloud ERP Solution Right for You? Strategic finance, softresources, 2013

[18] Staehr, L., Understanding the role of managerial agency in achieving business benefits from ERP systems. Information Systems Journal, Vol. 20 Issue 3, pp 213-238, 2010

[19] Warren Kleinsmith, The Evolution to the Cloud -Are Process Theory Approaches for ERP Implementation Lifecycles Still Valid?. Business Systems Review, Vol. 2, Issue 3, 2013 